\title{
Linear and conic scalarizations for obtaining properly efficient solutions in multiobjective optimization
}

\author{
Masoud Karimi ${ }^{1} \cdot$ Balal Karimi $^{2}$
}

Received: 13 January 2017/Accepted: 4 August 2017/Published online: 28 August 2017

(c) The Author(s) 2017. This article is an open access publication

\begin{abstract}
The existence of equivalent scalar problems for properly efficient point of a given multiobjective optimization problem over arbitrary cones is studied by so many authors. This paper emphasizes two scalarizations, i.e., linear scalarization and conic scalarization, and studies geometrical viewpoint on the relationship between proper efficiency and these scalarizations. We also show that conic scalarization is a generalization of linear scalarization based on augmented dual cone which provides a new type of trade-off for properly efficient solutions.
\end{abstract}

Keywords Multiobjective optimization - Proper efficiency $\cdot$ linear scalarization $\cdot$ conic scalarization

\section{Introduction}

Multiobjective optimization is an area of multiple criteria decision making, that is concerned with mathematical optimization problems involving more than one objective function to be optimized simultaneously. Multiobjective optimization has been applied in many fields of science, including engineering, economics and logistics where optimal decisions need to be taken in the presence of tradeoffs between two or more conflicting objectives [4, 15].

Masoud Karimi

karimi.masoud1984@gmail.com; karimi.masoud@razi.ac.ir

Balal Karimi

b.karimi1987@gmail.com

1 Department of Mathematics, Faculty of Science, Razi University, Kermanshah, Iran

2 Department of Mathematics, Karaj Branch, Islamic Azad University, Karaj, Iran
Minimizing cost while maximizing comfort while buying a car, and maximizing performance whilst minimizing fuel consumption and emission of pollutants of a vehicle are examples of multiobjective optimization problems involving two and three objectives, respectively. Generally, a multiobjective optimization problem is as follows:

$\min _{y \in Y \subset \mathbb{R}^{p}} y$,

where $Y \subset \mathbb{R}^{p}$ is the feasible set in objective space.

In this problem, we are looking for solutions in which improving in any objective function leads to impairing one of the others. These solutions are called (Pareto) efficient solutions. However, this is not always appropriate and this set of solutions is generally restricted to avoid some undesirable situations. These better solutions are named properly efficient solutions which were firstly described by Kuhn and Tucker in which the faults of the notion of efficiency were presented and the relations of the properly efficient solutions to scalarization problems were investigated [14]. Later Geoffrion defined proper efficiency by eliminating unbounded trade-offs between objectives and studied their relation to Kuhn and Tucker's proper efficiency and linear scalarization [6]. This concept was generalized by Borwein [2,3] and Benson [1] to problems that the objective space is ordered by closed convex cones. There exist so many definitions and interpretations of proper efficiency, so we refer to the papers $[7-9,16]$. We start with definitions of efficiency and proper efficiency.

Definition 1 [4] Consider the vector optimization problem (1). The feasible solution $\hat{y} \in \mathbb{R}^{p}$ is said to be an (Pareto) efficient element of (1) if

$(Y-\hat{y}) \cap\left(-\mathbb{R}_{+}^{p} \backslash\left\{0_{\mathbb{R}^{p}}\right\}\right)=\emptyset$. 
Geoffrion [6] defined proper efficiency in the following form.

Definition 2 [6] An efficient point $\hat{y} \in Y$ is called a properly efficient solution in Geoffrion's sense if there exists a positive real number $M$ such that for any $y \in Y$, $i \in\{1, \ldots, p\}$ with $y_{i}<\hat{y}_{i}$ there is an index $j \in\{1, \ldots, p\}$ with $\hat{y}_{j}<y_{j}$ such that

$\frac{\hat{y}_{i}-y_{i}}{y_{j}-\hat{y}_{j}} \leq M$.

Benson [1] defined proper efficiency as follows.

Definition 3 [1] The feasible element $\hat{y} \in \mathbb{R}^{p}$ is said to be a properly efficient element in Benson's sense of (1) if

$\operatorname{cl}\left(\operatorname{cone}\left(Y+\mathbb{R}_{+}^{p}-\hat{y}\right)\right) \cap\left(-\mathbb{R}_{+}^{p} \backslash\left\{0_{\mathbb{R}^{p}}\right\}\right)=\emptyset$.

Scalarizing a multiobjective optimization problem means formulating a single-objective optimization problem such that optimal solutions to the single-objective optimization problem are Pareto efficient solutions to the multiobjective optimization problem. In addition, it is often required that every Pareto efficient solution can be reached with some parameters of the scalarization. With different parameters for the scalarization, different Pareto efficient points are produced. A general formulation for a scalarization of a multiobjective optimization is thus

$\min _{y \in Y_{\theta}} g(y, \theta)$.

where $\theta$ is a vector parameter, the set $Y_{\theta} \subseteq Y$ is a set depending on the parameter $\theta$ and $g: \mathbb{R}^{p+1} \mapsto \mathbb{R}$ is a function.

Very well-known examples are the so-called linear scalarization, achievement scalarizing problems of Wierzbicki, $\epsilon$-constraint method. A new scalarization method is conic scalarization proposed by Kasimbeyli [11] which is a generalization of linear scalarization. This popular scalarization derives from conic separation theorem proposed by Kasimbeyli [12] which separates two closed (not necessarily convex) cones, analytically (see the Definition 5 and the Theorem 3). Conic scalarization uses some elements of augmented dual cone for scalarizing, in contrast with linear scalarization which uses some elements of dual cone in dual space. This elements produce a cone in place of a hyperplane for scalarizing. This paper considers two linear scalariztion and conic scalarization for determining properly efficient solutions. The paper also interprets these two scalarizations, geometrically. Using Hahn-Banach separation theorem and conic separation theorem, we show that conic scalarization is a generalization of linear scalarization which uses Bishop-Phelps cone for separating and scalarizing. By using conic scalarization, we will also propose a new formulation of trade-off for defining of properly efficient points based on conic scalarization.

The paper is organized as follows: In the Sect. 2 the relationship between linear scalarization and proper efficiency will be studied, geometrically. The interpretation of conic scalarization will be investigated in the Sect. 3. In this section, first the separation theorem for an arbitrary two closed cones $K$ and $C$ is given. Then, conic scalarization and new definition of trade-off are proposed. Finally, the last section presents some conclusions.

\section{Linear scalarization}

The Hahn-Banach Theorem is a central tool in functional analysis. It allows the extension of bounded linear functionals defined on a subspace of some vector space to the whole space, and it also shows that there are a sufficient number of continuous linear functionals defined on every normed vector space to make the study of the dual space. Another version of Hahn-Banach theorem is known as the Hahn-Banach separation theorem, and has numerous uses in convex analysis [17]. The following theorem is Hahn-Banach separation theorem based on two closed convex cones.

Theorem 1 If $K$ and $C$ are two closed convex cones and $K \cap C=\left\{0_{\mathbb{R}^{n}}\right\}$, then there exists a nonzero vector $y^{*} \in R^{n}$ such that, the hyperplane $\left\{y \in R^{n}:\left\langle y^{*}, y\right\rangle=0\right\}$ separates $K$ and $C$ and hence the following inequalities are satisfied:

$\left\langle y^{*}, d\right\rangle<0 \leq\left\langle y^{*}, y\right\rangle, \quad$ forall $y \in K$ and $d \in C \backslash\left\{0_{\mathbb{R}^{n}}\right\}$.

In the rest of this section, the relationship between linear scalarization and properly efficient points will be mentioned. The motivation for introducing proper efficient points is that it enables one to prove the existence of equivalent scalar problems whose solutions produce at least most of the efficient points, namely the proper ones [2].

If $y^{*} \in \mathbb{R}_{++}^{p}$ and $Y$ is a nonempty subset of $\mathbb{R}^{p}$, consider the problem

$\min _{y \in Y}\left\langle y^{*}, y\right\rangle$,

where the $y^{*}$ is often normalized according to $\sum_{i=1}^{p} y_{i}^{*}=1$. Hurwicz [10] showed that optimal solution of the linear scalarization is a properly efficient solution and also Borwein [2] showed that if $Y$ is a starshaped set at the properly efficient point $\hat{y}$, then there exists a positive vector $\hat{y}^{*} \in \mathbb{R}^{p}$ such that

$0 \leq\left\langle\hat{y}^{*}, y-\hat{y}\right\rangle, \quad$ forall $y \in Y$

i.e., $\hat{y}$ is an optimal solution of the linear scalarization with the positive weighted vector $\hat{y}^{*}$, see the Figs. 1 and 2 .

Let $\hat{y}$ be a properly efficient solution of (1) and let $y^{*}$ be a positive weighted vector for obtaining $\hat{y}$. Because $\hat{y}$ is a 
properly efficient solution, for any $y \in Y$ and for any $i \in$ $\{1,2, \ldots, p\}$ with $y_{i}<\hat{y}_{i}$ there is an index $j \in\{1,2, \ldots, p\}$ with $\hat{y}_{j}<y_{j}$. Therefore, assume that $y \in Y$ and $i \in$ $\{1,2, \ldots, p\}$ satisfy $y_{i}<\hat{y}_{i}$. There is an index $j \in$ $\{1,2, \ldots, p\}$ with $\hat{y}_{j}<y_{j}$ such that

$y_{i}^{*}\left(\hat{y}_{i}-y_{i}\right) \leq y_{j}^{*}\left(y_{j}-\hat{y}_{j}\right)$

$\Rightarrow \frac{\hat{y}_{i}-y_{i}}{y_{j}-\hat{y}_{j}} \leq \frac{y_{j}^{*}}{y_{i}^{*}}$,

because for all $y \in Y$

$\sum_{k=1}^{p} y_{k}^{*} \hat{y}_{k} \leq \sum_{k=1}^{p} y_{k}^{*} y_{k}$.

Thus, $\frac{y_{j}^{*}}{y_{i}^{*}}$ can be a substitute for $M$ in Geoffrion's definition of proper efficiency if $y^{*}$ is a positive weighted vector.

\section{Conic scalarization}

The conic scalarization method proposed by Kasimbeyli [11] for scalarization of nonconvex vector optimization problems. He introduced a special class of monotonically increasing sublinear scalarizing functions and showed that the zero sublevel set of every function from this class is a convex closed and pointed cone which contains the negative ordering cone [11]. Using the notion of a separable cone and conic separation theorem (see Theorem 3), Kasimbeyli introduced the conic scalarization method for scalarizing of nonconvex vector optimization problems. Kasimbeyli showed that him scalar optimization problem enables to characterize the complete set of efficient and properly efficient solutions of vector optimization problems without convexity and boundedness conditions $[5,11,12]$. In this section, we will first propose some preliminary of augmented dual cone and conic separation

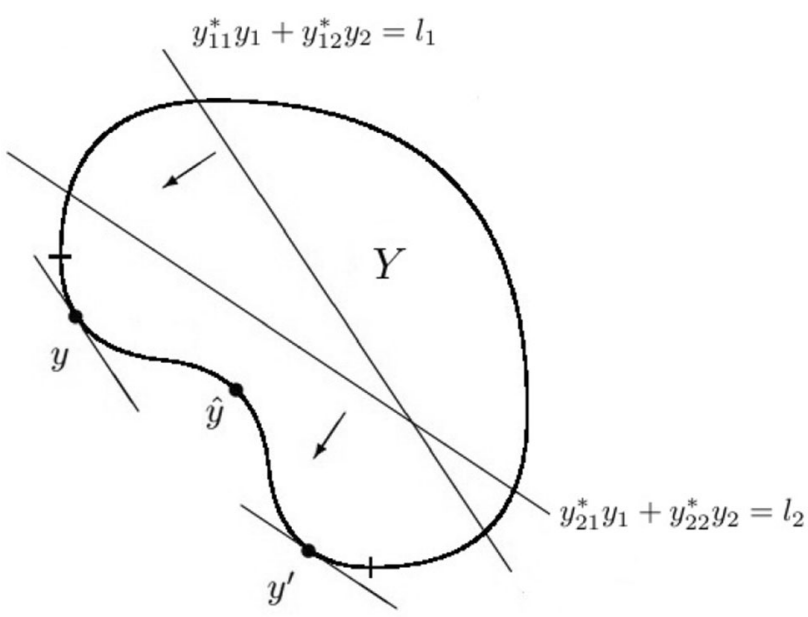

Fig. $1 Y$ is a nonconvex set and there exist no $y^{*}$ to obtain $\hat{y}$

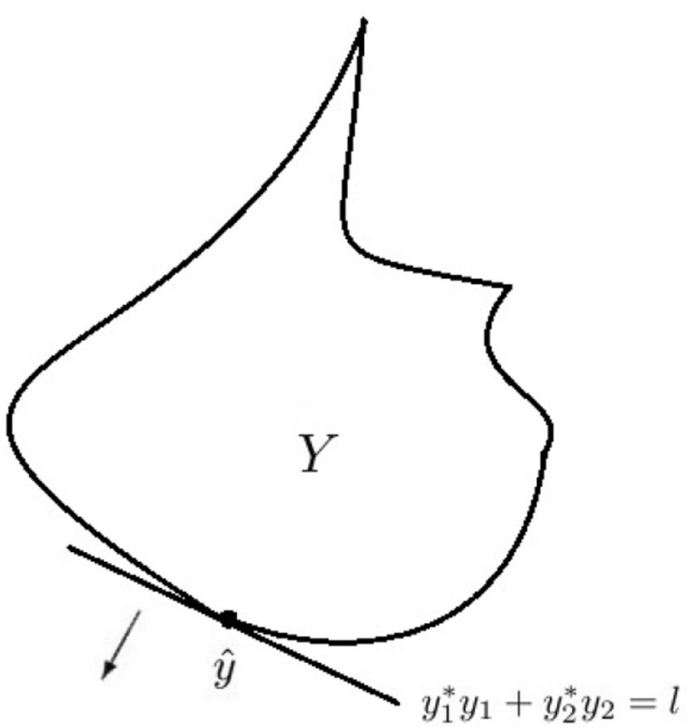

Fig. $2 Y$ is a starshaped set at the properly efficient points $\hat{y}$ and there exists some $y^{*}$ to obtain $\hat{y}$

theory proposed by Kasimbeyli [12] in Euclidian space $\mathbb{R}^{p}$. Then, the conic scalarization method will be interpreted.

\section{Conic separation theorem}

This subsection is devoted to some concept of augmented dual cone and conic separation theory proposed by Kasimbeyli [12]. Consider $\mathbb{R}^{p}$ with the norm $l_{1}$ and the ordering cone $\mathbb{R}_{+}^{p}$. In throughout of this paper, the notation $\|\cdot\|$ denotes the norm $l_{1}$.

The unit sphere and unit ball of $\Psi$ are denoted by

$U=\left\{y \in \mathbb{R}^{p}:\|y\|=1\right\}$

$B=\left\{y \in \mathbb{R}^{p}:\|y\| \leq 1\right\}$

A nonempty subset $C$ of $\mathbb{R}^{p}$ is called a cone if

$y \in C, \quad \lambda \geq 0 \Rightarrow \lambda y \in C$.

$\mathrm{C}$ is a pointed cone if

$C \cap-C=\left\{0_{\mathbb{R}^{p}}\right\}$.

cone $(S)=\{\lambda s: \lambda \geq 0$ and $s \in S\}$

$\operatorname{co}(S)$ denote the cone generated and convex hull by a set $S$, respectively.

$C_{U}=C \cap U=\{y \in C:\|y\|=1\}$

denotes the base norm of the cone $C$. Recall that the dual cone $C^{*}$ of $C$ and its quasi-interior $C^{\#}$ are defined by

$C^{*}=\left\{y^{*} \in \mathbb{R}_{+}^{p}:\left\langle y^{*}, y\right\rangle \geq 0\right.$ forall $\left.y \in C\right\}$,

and 
$C^{\#}=\left\{y^{*} \in \mathbb{R}_{+}^{p}:\left\langle y^{*}, y\right\rangle>0\right.$ forall $\left.y \in C \backslash\left\{0_{\mathbb{R}^{p}}\right\}\right\}$,

respectively. The following two cones called augmented dual cones of $C$ were introduced in $[11,12]$.

$C^{a *}=\left\{\left(y^{*}, \alpha\right) \in C^{\#} \times \mathbb{R}_{+}:\left\langle y^{*}, y\right\rangle-\alpha\|y\| \geq 0\right.$ forall $\left.y \in C\right\}$,

and

$C^{a \#}=\left\{\left(y^{*}, \alpha\right) \in C^{\#} \times \mathbb{R}_{+}:\left\langle y^{*}, y\right\rangle-\alpha\|y\|>0\right.$ forall $\left.y \in C \backslash\left\{0_{\mathbb{R}^{p}}\right\}\right\}$.

Kasimbeyli [11] shows that if $C=\mathbb{R}_{+}^{p}$, then

$$
\begin{aligned}
C^{a *}= & \left\{\left(y^{*}, \alpha\right) \in \mathbb{R}^{p+1}: 0 \leq \alpha \leq y_{i}^{*}, \text { forall } i=1, \ldots, p\right. \\
& \text { andthereexists } \left.k \text { suchthat } y_{k}^{*}>\alpha\right\}
\end{aligned}
$$

and

$$
C^{a \#}=\left\{\left(y^{*}, \alpha\right) \in \mathbb{R}^{p+1}: 0 \leq \alpha<y_{i}^{*} \text {, forall } i=1, \ldots, p\right\} .
$$

Definition 4 [12] The function $h$ on $Y \subset \mathbb{R}^{p}$ is called strongly monotonically increasing if, for each $y_{1}, y_{2} \in \mathbb{R}^{p}$, $y_{1}-y_{2} \in \mathbb{R}_{+}^{p} \backslash\left\{0_{\mathbb{R}^{p}}\right\} \Rightarrow h\left(y_{1}\right)>h\left(y_{2}\right)$.

Theorem 2 [12] Let $y^{*} \in \mathbb{R}_{+}^{p} \backslash\left\{0_{\mathbb{R}^{p}}\right\}$ and $\alpha \in \mathbb{R}_{+}$, and let function $h_{\left(y^{*}, \alpha\right)}: \mathbb{R}^{p} \rightarrow \mathbb{R}$ be defined as

$h_{\left(y^{*}, \alpha\right)}(y)=\left\langle y^{*}, y\right\rangle+\alpha\|y\|$.

Then, the function $h_{\left(y^{*}, \alpha\right)}$ is strongly monotonically increasing on $\mathbb{R}^{p}$ if and only if $\left(y^{*}, \alpha\right) \in\left(\mathbb{R}_{+}^{p}\right)^{a \#}$.

A general sufficient condition for two cones to satisfy the separation property is proved in [12].

Definition 5 (Separation property [12]) Let $C$ and $K$ be two closed cones of a $\mathbb{R}^{p}$ with base norms $C_{U}$ and $K_{U}$, respectively. Let $K_{U}^{\partial}=K_{U} \cap b d(K)$, and let $\tilde{C}$ and $\tilde{K}^{\partial}$ be the closures of the sets $\operatorname{co}\left(C_{U}\right)$ and $\operatorname{co}\left(K_{U}^{\partial} \cup\left\{0_{\mathbb{R}^{p}}\right\}\right)$, respectively. The cones $C$ and $K$ are said to have the separation property with respect to the norm $\|\cdot\|$ if

$\tilde{C} \cap \tilde{K}^{\partial}=\emptyset$.

The following theorem proved in [12] concern the existence of a pair $\left(y^{*}, \alpha\right) \in C^{a \#}$ for which the corresponding sublevel set $S\left(y^{*}, \alpha\right)$ of the strongly monotonically increasing sublinear function $h_{\left(y^{*}, \alpha\right)}(y)=$ $\left\langle y^{*}, y\right\rangle+\alpha\|y\|$ separates the given cones $C$ and $K$, where $S\left(y^{*}, \alpha\right)$ is defined as

$S\left(y^{*}, \alpha\right)=\left\{y \in \mathbb{R}^{p}:\left\langle y^{*}, y\right\rangle+\alpha\|y\| \leq 0\right\}$.

Theorem 3 (Conic separation theorem [12]) Let $C$ and $K$ be closed cones in the Euclidian space $\mathbb{R}^{p}$. Assume that the cones $-C$ and $K$ satisfy the separation property defined in Definition 5,

$-\tilde{C} \cap \tilde{K}^{\partial}=\emptyset$.

Then, $C^{a \#} \neq \emptyset$, and there exists a pair $\left(y^{*}, \alpha\right) \in C^{a \#}$ such that the corresponding sublevel set $S\left(y^{*}, \alpha\right)$ of the strongly monotonically increasing sublinear function $h_{\left(y^{*}, \alpha\right)}(y)=$ $\left\langle y^{*}, y\right\rangle+\alpha\|y\|$ separates the cones $-C$ and $b d(K)$ in the following sense:

$$
\left\langle y^{*}, y\right\rangle+\alpha\|y\|<0 \leq\left\langle y^{*}, z\right\rangle+\alpha\|z\|
$$

for all $y \in-C \backslash\left\{0_{\mathbb{R}^{p}}\right\}$, and $z \in b d(K)$. In this case the cone $-C$ is pointed. Conversely, if there exists a pair $\left(y^{*}, \alpha\right) \in$ $C^{a \#}$ such that the corresponding sublevel set $S\left(y^{*}, \alpha\right)$ of the strongly monotonically increasing sublinear function $h_{y^{*}, \alpha}(y)=\left\langle y^{*}, y\right\rangle+\alpha\|y\|$ separates the cones $-C$ and $b d(K)$ in the sense of (17) then the cones $-C$ and $K$ satisfy the separation property (16).

\section{Conic scalarization}

Consider the cone $\mathbb{R}_{+}^{p}$. From Theorem 3, for every proper efficient element $\hat{y}$ there exists a pair $\left(y^{*}, \alpha\right) \in\left(\mathbb{R}_{+}^{p}\right)^{a \#}$ such that for all $y \in Y$ and for all $c \in \mathbb{R}_{+}^{p} \backslash\left\{0_{\mathbb{R}^{p}}\right\}$, one has

$\left\langle y^{*},-c\right\rangle+s\|-c\|<0 \leq\left\langle y^{*}, y-\hat{y}\right\rangle+\alpha\|y-\hat{y}\|$.

Kasimbeyli in [11] showed that if there exists a pair $\left(y^{*}, \alpha\right) \in C^{a \#}$ such that

$\left\langle y^{*}, \hat{y}\right\rangle+\alpha\|\hat{y}\| \leq\left\langle y^{*}, y\right\rangle+\alpha\|y\|$,

for all $y \in Y$ then $\hat{y}$ is a properly efficient element of the optimization problem 1 . This property provides a conic scalarization for characterizing properly efficient elements of a nonconvex vector optimization problem; this characterization using conic scalarization proposed by Kasimbeyli [11]. Consider the augmented dual element $\left(y^{*}, \alpha\right)$ and define the following sublevel of zero

$S\left(y^{*}, \alpha\right)=\left\{y \in \mathbb{R}^{P}:\left\langle y^{*}, y\right\rangle+\alpha\|y\| \leq 0\right\}$.

Theorem 4 Let $\left(y^{*}, \alpha\right) \in\left(\mathbb{R}_{+}^{p}\right)^{a \#}$. Then,

$-\mathbb{R}_{+}^{p} \subset S\left(y^{*}, \alpha\right)$.

Proof Assume that $y$ is a nonzero element of $-\mathbb{R}_{+}^{p}$. From definition of $\left(\mathbb{R}_{+}^{p}\right)^{a \#}$, if $\left(y^{*}, \alpha\right) \in\left(\mathbb{R}_{+}^{p}\right)^{a \#}$ then $\left\langle y^{*},-y\right\rangle>\alpha\|-y\|$. Then $\left\langle y^{*}, y\right\rangle+\alpha\|y\|<0$. Since

$0_{\mathbb{R}^{p}} \in-\mathbb{R}_{+}^{p} \cap\left\{y \in \mathbb{R}^{P}:\left\langle y^{*}, y\right\rangle+\alpha\|y\| \leq 0\right\}$,

one has

$-\mathbb{R}_{+}^{p} \subset\left\{y \in \mathbb{R}^{P}:\left\langle y^{*}, y\right\rangle+\alpha\|y\| \leq 0\right\}$.

An geometric illustrative of Theorem 4 is given in Fig. 3. 


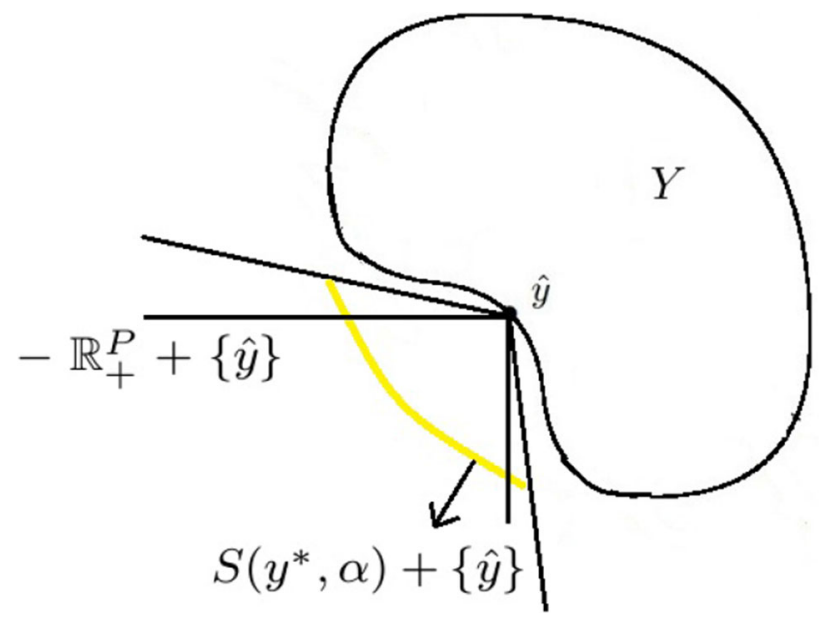

Fig. 3 The set $S\left(y^{*}, \alpha\right)+\{\hat{y}\}$ separates the properly efficient point $\hat{y}$ and the set $Y \backslash\{\hat{y}\}$

It is clear that $S\left(y^{*}, \alpha\right)$ is a closed convex cone. This is a known cone so-called Bishop-Phelps cone generated by augmented dual element $\left(-y^{*}, \alpha\right)$ and the norm $\|\cdot\|$. Kasimbeyli and Kasimbeyli [13] showed that if $K \cap$ $C \backslash\left\{0_{\mathbb{R}^{p}}\right\}=\emptyset$ where $K$ closed cone and $C$ is a BishopPhelps cone, then $K$ and $C$ satisfy the separation property and, hence, these two cones satisfy the conic separation Theorem 3 .

Theorem 5 [5] Let $\left(y^{*}, \alpha\right) \in\left(\mathbb{R}_{+}^{p}\right)^{a \#}$. Assume that $\hat{y}$ is a minimal element of the following problem

$\min _{y \in Y}\left\langle y^{*}, y\right\rangle+\alpha\|y\|$.

Then $\hat{y}$ is a properly efficient element of (1).

The Theorem 5 shows that if there exists some element $\left(y^{*}, \alpha\right) \in\left(\mathbb{R}_{+}^{p}\right)^{a \#}$, then the Bishop-Phelps cone $S\left(y^{*}, \alpha\right)$ separates $-\mathbb{R}_{+}^{p}$ and $(Y \backslash b d(Y))-\{\hat{y}\}$. Then, if the problem (18) has a bounded optimal solution $\hat{y} \in b d(Y)$ then this solution is a properly efficient point. On the other hand, it is clear that this scalarization generalizes the linear scalarization, setting $\alpha=0$ in the problem 18 .

Theorem $6[11,12]$ Assume that $\hat{y}$ is a properly efficient element of (1). Then, there exists $\left(y^{*}, \alpha\right) \in\left(\mathbb{R}_{+}^{p}\right)^{a \#}$ such that $\hat{y}$ is a minimal element of the following problem:

$\min _{y \in Y}\left\langle y^{*}, y-\hat{y}\right\rangle+\alpha\|y-\hat{y}\|$.

The Theorem 6 shows that if the point $\hat{y} \in Y$ is a properly efficient element, then there exists some augmented dual element $\left(y^{*}, \alpha\right) \in\left(\mathbb{R}_{+}^{p}\right)^{a \#}$ such that the sublevel $S\left(y^{*}, \alpha\right)$ separates $-\mathbb{R}_{+}^{p}$ and $(Y \backslash b d(Y))-\{\hat{y}\}$.
Theorem 7 Assume that $\hat{y}$ is a properly efficient element of (1). Then, there exists a sublevel cone $S\left(y^{*}, \alpha\right)$ such that $S\left(y^{*}, \alpha\right)$ separates $-\mathbb{R}_{+}^{p}$ and $(Y \backslash b d(Y))-\{\hat{y}\}$.

Proof Assume that $\hat{y}$ is a properly efficient element of (1). Then,

$\operatorname{cl}\left(\operatorname{cone}\left(Y+\mathbb{R}_{+}^{p}-\hat{y}\right)\right) \cap\left(-\mathbb{R}_{+}^{p}\right) \backslash\left\{0_{\mathbb{R}^{p}}\right\}=\emptyset$.

Because $\mathbb{R}_{+}^{p}$ with respect to the norm $l_{1}$ is a Bishop-Phelps cone, and from Theorem 3 there exists some element $\left(y^{*}, \alpha\right) \in\left(\mathbb{R}_{+}^{p}\right)^{a \#}$ such that

$\left\langle y^{*}, c\right\rangle+\alpha\|c\|<0 \leq\left\langle y^{*}, y+d-\hat{y}\right\rangle+\alpha\|y+d-\hat{y}\|$,

for all $c \in-b d\left(\mathbb{R}_{+}^{p}\right), d \in \mathbb{R}_{+}^{p}$ and $y \in Y$. This means the zero sublevel $S\left(y^{*}, \alpha\right)$ separates $-\mathbb{R}_{+}^{p}$ and $(Y \backslash b d(Y))-\{\hat{y}\}$.

Theorem 8 [11] Consider the multiobjective optimization problem 1 . Then,

(i) Assume that $\hat{y}$ is a properly efficient element of (1). Then, there exist $\left(y^{*}, \alpha\right) \in\left(\mathbb{R}_{+}^{p}\right)^{a \#}$ and $r \in \mathbb{R}^{p}$ such that $\hat{y}$ is a minimal element of the following problem:

$$
\min _{y \in Y}\left\langle y^{*}, y-r\right\rangle+\alpha\|y-r\| \text {. }
$$

(ii) If there exist $\left(y^{*}, \alpha\right) \in\left(\mathbb{R}_{+}^{p}\right)^{\text {a\# }}$ and $r \in \mathbb{R}^{p}$ such that $\hat{y}$ is a minimal element of the problem (19), then $\hat{y}$ is a properly efficient element of the multiobjective optimization problem (1).

Let $\hat{y}$ be a properly efficient solution of (1) and let $\left(y^{*}, \alpha\right) \in\left(\mathbb{R}_{+}^{p}\right)^{a \#}$ be a positive weighted vector for obtaining $\hat{y}$. Because $\hat{y}$ is a properly efficient solution, for any $y \in Y$ and for any $i \in\{1,2, \ldots, p\}$ with $y_{i}<\hat{y}_{i}$ there is an index $j \in\{1,2, \ldots, p\}$ with $\hat{y}_{j}<y_{j}$. Therefore, assume that $y \in Y$ and $i \in\{1,2, \ldots, p\}$ satisfy $y_{i}<\hat{y}_{i}$. There is an index $j \in\{1,2, \ldots, p\}$ with $\hat{y}_{j}<y_{j}$ such that

$$
\begin{aligned}
& y_{i}^{*} \hat{y}_{i}+y_{j}^{*} \hat{y}_{j}+\alpha\left|\hat{y}_{i}\right|+\alpha\left|\hat{y}_{j}\right| \leq y_{i}^{*} y_{i}+y_{j}^{*} y_{j}+\alpha\left|y_{i}\right|+\alpha\left|y_{j}\right| \\
& \quad \Rightarrow y_{i}^{*}\left(\hat{y}_{i}-y_{i}\right)+\alpha\left(\left|\hat{y}_{i}\right|-\left|y_{i}\right|\right) \leq y_{j}^{*}\left(y_{j}-\hat{y}_{j}\right)+\alpha\left(\left|y_{j}\right|-\left|\hat{y}_{j}\right|\right) \\
& \quad \Rightarrow \frac{\left(\hat{y}_{i}-y_{i}\right)+\frac{\alpha}{y_{i}^{*}}\left(\left|\hat{y}_{i}\right|-\left|y_{i}\right|\right)}{\left(y_{j}-\hat{y}_{j}\right)+\frac{\alpha}{y_{j}^{*}}\left(\left|y_{j}\right|-\left|\hat{y}_{j}\right|\right)} \leq \frac{y_{j}^{*}}{y_{i}^{*}} .
\end{aligned}
$$

Based on above-mentioned, we proposed a new definition of proper efficiency based on trade-off for nonconvex problem as follows:

Definition 6 An efficient solution $\hat{y} \in Y$ is called a properly efficient solution if there exist a positive real number $M$ and nonnegative numbers $s_{1}, s_{2}$ such that for 
Table 1 Comparing linear and conic scalarizations

\begin{tabular}{lll}
\hline Reference & Linear scalarization & Conic scalarization \\
\hline Origin & Hahn-Banach theorem & Conic separation theorem \\
Weighted vector origin & Dual cone & Augmented dual cone \\
Formulation & $\left\langle y^{*}, y\right\rangle$ & $\left\langle y^{*}, y\right\rangle+\alpha\|y\|$ \\
Usable & Convex problem & Nonconvex problem \\
Geometrical form & Hyperplane & Cone \\
Trade-off form & $\frac{\hat{y}_{i}-y_{i}}{y_{j}-\hat{y}_{j}} \leq M$ & $\frac{\left(\hat{y}_{i}-y_{i}\right)+s_{1}\left(\left|\hat{y}_{i}\right|-\left|y_{i}\right|\right)}{\left(y_{j}-\hat{y}_{j}\right)+s_{2}\left(\left|y_{j}\right|-\left|\hat{y}_{j}\right|\right)} \leq M$ \\
\hline
\end{tabular}

any $y \in Y$ and for any $i \in\{1, \ldots, p\}$ with $y_{i}<\hat{y}_{i}$ there is an index $j \in\{1, \ldots, p\}$ with $\hat{y}_{j}<y_{j}$ such that

$$
\frac{\left(\hat{y}_{i}-y_{i}\right)+s_{1}\left(\left|\hat{y}_{i}\right|-\left|y_{i}\right|\right)}{\left(y_{j}-\hat{y}_{j}\right)+s_{2}\left(\left|y_{j}\right|-\left|\hat{y}_{j}\right|\right)} \leq M \text {. }
$$

Theorem 9 Let $\hat{y} \in Y$ be a properly efficient solution in sense Geoffrion definition. Then, $\hat{y} \in Y$ satisfies Definition 6.

Proof Let $\hat{y} \in Y$ be a properly efficient solution in sense Geoffrion definition. Setting $s_{1}=s_{2}=0$. It is clear that $\hat{y} \in Y$ satisfies Definition 6 .

This theorem shows that the new definition of properly efficient generalizes Geoffrion definition of properly efficient.

Table 1 is presented for comparing linear scalarization and conic scalarization.

As is mentioned, Table 1 compares two linear and conic scalarization together. The first row for the table shows the origin of these scalarizations. Linear scalarization is originated from Hahn-Banach separation theorem which separates two convex sets. Conic scalarization is originated from Kasimbeyli's conic separation theorem which separates two closed cones. Kasimbeyli and Karimi have developed this type of separation theorem for two arbitrary nonconvex sets under some mild conditions. Second and third row show how these scalarizations use Hahn-Banach theorem and conic separation theorem. Forth row states the linear scalarization is compatible with convex problem, and the conic scalarization is compatible with nonconvex optimization problem. This is due to the fact that two separable convex sets will be separated using some hyperplane, and two separable nonconvex sets will be separated using some cones. Finally, last row of the table shows some fact about trade-off in convex and nonconvex multiobjective optimization problems.

\section{Conclusion}

This paper studies on the relationship between two scalarizations and proper efficiency. One of this scalarization is linear scalarization which is popular and known in multi-objective optimization and vector optimization. Linear scalarization originated from Hahn-Banach separation theorem for separating two convex sets. For determining properly efficient points in a convex multiobjective optimization problem, linear scalarization is a useful tool. But, in nonconvex multiobjective optimization problem there exist no guarantee to existence dual elements for determining properly efficient solutions. Kasimbeyli in [12] proposed a conic separation theorem for separating two nonconvex cones and this separation theorem provides conic scalarization to scalarize nonconvex optimization problems. In contrast with linear scalarization, conic scalarization considers a given Bishop-Phelps cone instead of a given a hyperplane, geometrically. In this paper, we also propose a new definition of proper efficiency which has obtained conic scalarization. This new definition of proper efficiency provide a new trade-off of a given properly efficient solution which can be new area of research.

Open Access This article is distributed under the terms of the Creative Commons Attribution 4.0 International License (http://crea tivecommons.org/licenses/by/4.0/), which permits unrestricted use, distribution, and reproduction in any medium, provided you give appropriate credit to the original author(s) and the source, provide a link to the Creative Commons license, and indicate if changes were made.

\section{References}

1. Benson, H.P.: An improved definition of proper efficiency for vector maximization with respect to cones. J. Math. Anal. Appl 71, 232-241 (1979)

2. Borwein, J.M.: The geometry of Pareto efficiency over cones. Optimization 11, 235-248 (1980)

3. Borwein, J.M.: Proper efficient points for maximizations with respect to cones. SIAM J. Control Optim 15, 57-63 (1977)

4. Ehrgott, M.: Multicriteria Optimization. Springer, Berlin (2005)

5. Gasimov, R.N.: Characterization of the Benson proper efficiency and scalarization in nonconvex vector optimization. In: Köksalan, M., Zionts, S. (eds.) Multiple Criteria Decision Making in the New Millennium: Proceedings of the Fifteenth International Conference on Multiple Criteria Decision Making (MCDM) Ankara, Turkey, July 1014, 2000, Vol. 507, pp. 189-198. Springer Science \& Business Media (2000) 
6. Geoffrion, A.M.: Proper efficiency and the theory of vector maximization. J. Math. Anal. Appl 22, 618-630 (1968)

7. Guerraggio, A., Molho, E., Zaffaroni, A.: On the notion of proper efficiency in vector optimization. J. Optim. Theory Appl 82, 1-21 (1994)

8. Hartley, R.: On cone-efficiency, cone-convexity and cone-compactness. SIAM J. Appl. Math 34, 211-222 (1978)

9. Henig, M.I.: Proper efficiency with respect to cones. J. Optim. Theory Appl. 36, 387-407 (1982)

10. Hurwicz, L.: Programming in linear spaces. In: Arrow, K.J., Hurwicz, L., Uzawa, H. (eds.) Studies in Linear and Nonlinear Programming. Stanford University Press, Stanford (1958)

11. Kasimbeyli, R.: A conic scalarization method in multi-objective optimization. J. Glob. Optim. 56, 279-297 (2013)

12. Kasimbeyli, R.: A nonlinear cone separation theorem and scalarization in nonconvex vector optimization. SIAM J. Optim. 20, 1591-1619 (2013)
13. Kasimbeyli, N., Kasimbeyli, R.: A representation theorem for Bishop-Phelps cones. Pac. J. Optim. 13(1), 55-74 (2017)

14. Kuhn, H., Tucker, A.: nonlinear programming. In: Neyman, J. (ed.) Proceedings of the Second Berkeley Symposium on Mathematical Statistics and Probability, pp. 481-492 (1951)

15. Miettinen, K.: Nonlinear Multiobjective Optimization. Springer, Berlin (1999)

16. Pourkarimi, L., Karimi, M.: Characterization of substantial and quasi-substantial efficient solutions in multiobjective optimization problems. Turk. J. Math. 41, 293-304 (2017)

17. Rockafellar, R.T.: Convex Analysis. Princeton University Press, Princeton (1970) 